dieser Concretionen bei den Herbivaren rücksictitlich der BEstandtheile constant ist.

\author{
Zweite Abtheilung. \\ Naturgeschichte und Pharmakognosie.
}

\title{
Ueber gediegen Gold
} ron

\section{G. $\boldsymbol{R} \circ \boldsymbol{s} e$}

Eine sehr ausführliche Reihe von Untersuchungen über das gediegene Gold, besonders des Goldes vom Ural ist von $\mathrm{Hr}$. Bose unternommen und in Poggendorfts Ann. B XXul. S. 161. behannt gemacht worden. Aus den Untersuchungen von Klaproth, insbesondere aber aus den neueren von Boussingault ergiebt sich: dafs in dem gediegenen Golde seine Bestandtbeile, Gold und Silber, in bestimmten Proportionen vorkommen. Indessen hat die Ansicht, dafs das gediegene Gold nach bestimmten Proportionen zusammengesetzt sey, etwas Eefremdendes. Die Krystallformen des Goldes und Silbers stimmen so sehr mit einander uberein, dafs man geneigt ist, diese Metalle für isomorph zu halten; aber isomorphe Substanzen sind gewöhnlich nicht nach bestimmiten Proportionen mit einander verbunden. Es wäre eben so auffallend, Antimou, Arsenili und Tellur in bestimmten Verhältnissen mit einander verbunden zu sehen. Da indessen isomorphe Hörper auch aach bestimmten Verhältnissen zusammengesetzt rortiommen, so sind die Boussingault'schen Analysen nur darin auffallend, dafs nach densetben Gold und Silber stets in bestimmten Verhältnissen verbunden sind. 
Das Gold am Ural kommt theils anstehend theils lose im Sande als Waschgold. vor. Vor der Fntdeclung des letztern, 1819 , wurde das Gold nur durch unterirdischen Bergbau gewonnen. Seit dieser Zeit aber hat man den wenig lohnenden Bergbau größstentheils eingestellt. Wo das Gold ansteht, lömmt es auf Gängen von Quarz vor; das von Beresow findet sich hrystallisirt und derb in kleinen Parthieen; das von Neviansh kommt nicht krystallisirt, sondern nur in ein und aufgewachsenen Blättchen vor. Im Goldsand findet sich das Gold meistens in kleinen lkörnern und Sehüppchen. zuweilen liommen indessen Stiicke von beileutender Grölse vor, besonders in einer Wäsche Czarewo Mlexan. drowsh, bei Miash im südlichen Ural, wo Stücke von $13-$ 16 , selbst eins von $24^{2}, 3$ russischen Pfunden vorgekommen sind (s. A. r. Humboldt in Poggend. Ann. XVIII. 273). In dem Waschgolde finden sich auch Heine Krystalle von Gold. die zwar heine scharfen Kanten und Ecken haben, doch hinlänglich deutlich ihre Form erkenzen lassen.

Das Gold vom Ural enthält au'ser dem Silher noch Spuren von Eisen und Kupfer. Da Gold und Platin am Ural häufí auf denselben Lagerstätten vorkommen, so wäre es wohl müglich, daf's diese Metalle auch chemisch mit einander verhunden wären. G. Rose untersuchte deshalb besonders in dieser Rüclisicht Gold von zwanzig versehicdenen Lager. stätten, aber weder Osmium, noch Platin, Iridium noch Palladium Lonnten darin entdecht werden. Es ist behannf; dafs man schon Verbindungen von Rhodium. sowoin als Pailadiun mit Gold gefunden hat, dafs del Rio die erste Verbindung in dem mexikanischen Golde, and Cloud in Philadelphia die zweite in Goldbarren gefunden hat, die aus Brasilien gehommen waren, weshalb G. Aose auch besonders seine Aufinerksamkeit anf diese Metalle richtete.

Die Analysen reigten nun, daf's der Siibergehait in einem 
selr abweichenden Verhältnifs in dem gediegenen Golde vorkomme; wir wollen aus den zahlreichen Analysen nur einige Beispiele anfithren. Das von Schabrowshi bei Katharinenburg enchielt 0,16 , das von Boruschka bei Nischno Tagil 5,23, das von Bernsow 7,02, cin anderes ron Boruschka 9,02, wieder cin anderes 16,15 , das von Siranowsti in Altai $38,38 \%$, Silber.

Die sämmtlichen von G. Rose angesteliten Analysen ergaben als Resultat, dars bei dem natürlich vorkommenden Golde an eine Verbindung von Gold und Silber nach natïrlichen Proportionen nicht zu denken sey. Scheint diesus auch bei einigen Körnern der Fall zu seyn, so ist doch dic Mehrzahl damit gar nicht in Uebereinstimmung zu bringen, und die rollständigen Uebergänge, die sich ron einem Ver. hältnifs zum andern finden, beweisen am besten, dafs die bestimnten Verhältnisse hier gar nicht existiren. Und dies ist eben so der Fall bei den Verbindungen, die viel Siller enthalten, wie bei denen die nur wenig Silber enthalten, bei denen die im Goldsande rorkonmen, als bei solchen, die anf der urspringlichen Iagerstätte selbst gebrochen sind.

Dieses Verbundenseyn des Goldes and Silbers in unbe. otimmten Verhältnissen, errriebt nun mit Bestimmtheit, dafs Gold und Silber isomorphe Körper sind. Aus der Betrachtung der Kustalifo:m des Goldes und Silbers war dieses Resultat nicht unmittelbar abzuleiten. Die Formen dieser Hétalle gehören zum regulären Krystallisationssysteme, und es ist bekannt, dufs Körper, die die verschiedenste ehemische Zusammensetzung haben, in eben diesen Formen krystallisiren; indessen machte doch die grofse Uebereinstimmung in den Formen ron Gold und Sither, unter lenen gewisse, die stanst beim regularren System häufig vorkommen, ganz ausgescblossen sind, und andere auf alcictse Wcise nur beim Gold ard Silber vorkommen, und die gleiche Suucturrerhültnisse 
des Goldes und Silbers den Isomorphismus dieser beiden Me. talle immer sehr wahrscheinlich. In einer folgenden Abbandlung (Poggend. Annal. XXIII. 186) hat G. Rose die Krystallformen des Goldes und Silbers einer genauen Untersuchung unterworfen.

Ueber die Methode, welche G. Rose bei den Analysex des Goldes befolgte, wollen wir noch Folgendes anführen. Sie war verschieden je nachlem das Gold mehr oder weniger Silber enthielt, welches schon an der messing- oder goldgelben Farbe zu erkennen ist.

Das goldgelbe Gold wuris ir: Künigswasser aufgelöst, das Silber blieb grölstentheils als Chlorsilber zurück und hatte noch die Form der angewandten Goldplatte. Die Aufösung wurde abgegossen, das Chlorsilber mit einem Glasstabe zer. drückt und aufs Neue mit lönigswasser behandelt. Beide Aufgïsse von Könnigswasser wurden mit Wasser verdïnnt, um das noch aufgelöste Chlorsilber abzuscheiden. Das sämmtliche Chlorsilher wurde gesammelt und sein Gewicht bestimmt. Die von Chlorsilber getrennte Aullösung $w$ rde in einer Por. zellanschale verdampft, der Rïclistand in $W$ asser aufgeweicht und mit Oxalsäure versetzt. Das Becherglas war mit einem convexen Deckglase bedecht worden, damit das Gold, welches mit der sich entwichelnden Kohlersäure mechanisch fortgerissen wurde, nicht verloren ging. Die neutrale Goldauflösung trübt sich beim Zusatz von Oxalsiure augenblistlich, die getrübte Flïsigkeit sieht in gebrochenen Iichte grün, im zurückgeworfenen braun und undurchsichtig aus; doch bedarf es noch einiger Zeit, ehe das Gold vollständig gefält ist, daher es 34 Stunden und länger auf der warmen Hapelle siehca gelassen wurde. Das Gold legte sich in metallischen Blättchen an die Wände des Glases an. Die Flüs. sigkeit ist voliständigg gefällt, renn sie ganz wasserklar ge. worden ist und sich knine Blasen, auch bei neuem Zusarz Ainal. d. Fliaru. Bd. Vil. Hefe: 
von Oxalsäure mehr entwicheln. Sie wurde dann fon dern ausgeschiedenen Golde abfitrirt, welches gewogen wurde, das Filtrat aber zu: 'Trockne verdampft und die überschüssig zugesetzte Oxalsäure verjagt. Es blieb nun immer ein kleiner: brauner Rüchstand, welcher Spuren von Hupfer und Fisen enthielt.

Wenn das gediegene fold gegen $20^{\circ}$ Silber fïlurt, so hat das bei der Behandlung mit Königswasser zurückbleibende Gold so viel Zusammenhalt, dals man unsicher ist, ob auch alles Siber in Chlorsilber verwandelt worden ist, und man hann diese Methode nur fïr den Fall anwenden, wenn man die Goldprobe zu einer sehr dïnnen Platte ausgeschlagen hat.

Das Gold, welches melor als 20\% Silber enthält, lifist sich also nicht gut nit Königswasser analysiren. Was gewöhnliche Verfahren es zu untersuchen, besteht darin, es an cupelliren, es mit Silber und Blei auf der Iapelle zusammen zu schmelzen, wobei sich las Blei in die Kapelle einzieht und eine J.egirung ron Gold und Silber zurüclibleibt, aus der sich nun das Silber mit Salpetersäure ausziehen lälst. Da indefs Gay-Lussac gezeigt hat, dafs dies Verfahren, auch bei der grölsten Vorsicht stets mit einem kleinen VerInst an Silber verbunden ist, so versuchte $G$. Rose noch einige andere Methaden; wir begnügen uns diejenige hier ansuführen, die den : wech an besten erreichte: nämlich das Gold wit Blei zu schmelzen.

Da Blei sehr leicht schmilzt und im geschmolzenen $\mathrm{Zu}$ stande Gold sehr leicht auflöst, so honnte die Znsammenschmelzung mit Gold schon in einem lileinen Porzellantiegel iiber der Spirituslampe mit doppeltem Luftzuge vorgenommen werden. Die geschmoizene Masse wurde im Tiegel mit Salpetersäure übergossen, nachdem sie sich losgelöst hatte, in

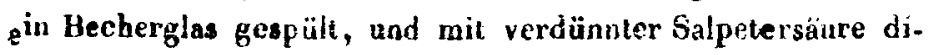
gerirt, welches nochmals wiederbolt wurde, un auch das 
salpetersaure Hleioxyd vïllig aufzulösen. Der unantigelöste Rüchstand wurde dann in Königswasser aufgelöst, dịt A ufö. sung mit Wasser verdïnnt, und das wenige Chlorsilber, was sich hier noch gebildet hatte, auf einem Filter gesammelt. Die filtrirte Flïssigkeit wurde zur Trockne verdamptt, der Rïchstand in Chlorwasserstoffsäure und Wasser aufgeiöst und das Gold mit einer Aullösung von Fisenchlorür gefällt. Ein: Aullösung von schwefelsaurem Eisenoxydul wurde vermieden, wreil das aufgelöste Gold vielleiclst noch Blei enthalten haben bonnte. Die salpetersaure Auflösung, welche zuerst orhalten war, wurde mit Wasser sehr verdünnt und darauf mit einer Auflösung von Chiorbici versetzt. Das Silber wurde nicht mit reiner Chlorwasserstoffsüure gefällt, um mit dem Cblorsilber sicht auch noch einen Niederschlag von Chlorblei za erhalten, - der bei seiner Schwerlösliehbeit sich nur schwer wirde haben wegwaschen lassen. Nachden* die Flüssigkeit warm gestellt $w a r$ wind sich vollkommen geldiirt hatte, wurde sie durch dasselbe Filter filtrirt, auf welches die Goldaullösung gegossen war. Die geringe Monge Eisen, welche noch in der durchgelaufenen Flüssigkeit war, konnte wegen der Nenge Blei nicht geschieden werden.

\title{
Ueber die Eermehrung der Blutegel
}

\author{
$\nabla 0 \mathrm{u}$ \\ Geisel $l e r$, \\ Apotheker su Königsberg in der Noumark.
}

Am 3 isten July 1832 erhielt ich von einem Blutegelfanger in hiesiger Gegend unter dem Namen Mutterblutegel einen Blut. egel, der sich ganz zusanmengezogen hatte und unter dessen Bauche zabllosie klcine weifse Fäden befindliós waren, die aur beim Bewegea des siẹ enthaltenden mit Wasser gefull. 